\title{
Qué tengo que saber para medir bien el ventrículo derecho por ecocardiograma
}

\author{
Javier Ibero Valencia \\ Isabel Ruiz Zamora \\ Luis Javier Alonso \\ Pedro María Azcárate
}

Correspondencia

Javier Íbero Valencia

jibero@riojasalud.es

Servicio de Cardiología. Hospital San Pedro. Logroño. La Rioja. España

Recibido: 28/01/2020

Aceptado: 28/02/2021

En línea: 30/04/2021

Citar como: Ibero Valencia J, Ruiz Zamora I, Alonso LJ, Azcárate PM. ¿Qué tengo que saber para medir bien el ventrículo derecho por ecocardiograma? Rev Ecocar Pract (RETIC). 2021 (Abril); 4 (1): 40-47. doi: 10.37615/retic.v4n1a2.

Cite this as: Ibero Valencia J, Ruiz Zamora I, Alonso LJ, Azcárate PM. What do I have to know to measure the right ventricle by echocardiogram? Rev Ecocar Pract (RETIC). 2021 (Abril); 4 (1): 40-47. doi: 10.37615/retic.v4n1a2

\section{Palabras clave}

$\triangleright$ Ventrículo derecho

$\triangleright$ Ecocardiograma

\begin{tabular}{l}
\hline Keywords \\
\hline$\triangleright$ Rigth ventricle \\
$\triangleright$ Echocardiography \\
\hline
\end{tabular}

\section{RESUMEN}

La dificultad técnica de la valoración del ventrículo derecho por medio de la imagen y, especialmente por ecocardiografía, hace que su evaluación quede relegada a un segundo plano en numerosas ocasiones. Este artículo de revisión se centra de forma específica en las particularidades del estudio del ventrículo derecho por ecocardiografía, sus indicaciones y limitaciones, así como el modo de interpretación de los principales parámetros empleados en la práctica clínica diaria.

ABSTRACT
Right ventricle echocardiographic evaluation is technically complex, affecting sometimes its quality in standard
studies. This review article focuses on how to properly measure and interpretate the most commonly use echo-
cardiographic parameters, taking into account its particularities, indications and limitations in our daily clinical
practice.

\section{Introducción}

A pesar de la relevancia clínica del ventrículo derecho (VD), la dificultad técnica de su valoración por medio de la imagen y, especialmente por ecocardiografía, hace que su evaluación quede relegada a un segundo plano en numerosas ocasiones. Por este motivo es necesario una estandarización del método de estudio con objeto de obtener la máxima información posible de esta compleja estructura. Este artículo de revisión se centra de forma específica en las particularidades del estudio del ventrículo derecho por ecocardiografía, sus indicaciones y limitaciones, así como el modo de interpretación de los principales parámetros empleados en la práctica clínica diaria.

\section{Anatomía y fisiología del ventrículo derecho}

\section{Anatomía del ventrículo derecho}

El VD es la estructura cardiaca más anterior y se encuentra inmediatamente posterior al esternón. Por lo tanto, su localización directamente detrás del esternón, el espesor de la pared del VD (2-5 mm) y su proximidad al transductor ecocardiográfico, hacen que en ocasiones sea un poco más complejo obtener ventanas adecuadas para una visualización clara y global del mismo. Además, su forma asimétrica, triangular en el plano coronal y de media luna en el transversal, hacen que sea necesaria la integración de diferentes imágenes obtenidas mediante diferentes ventanas para su visualización completa (Vídeo 1).

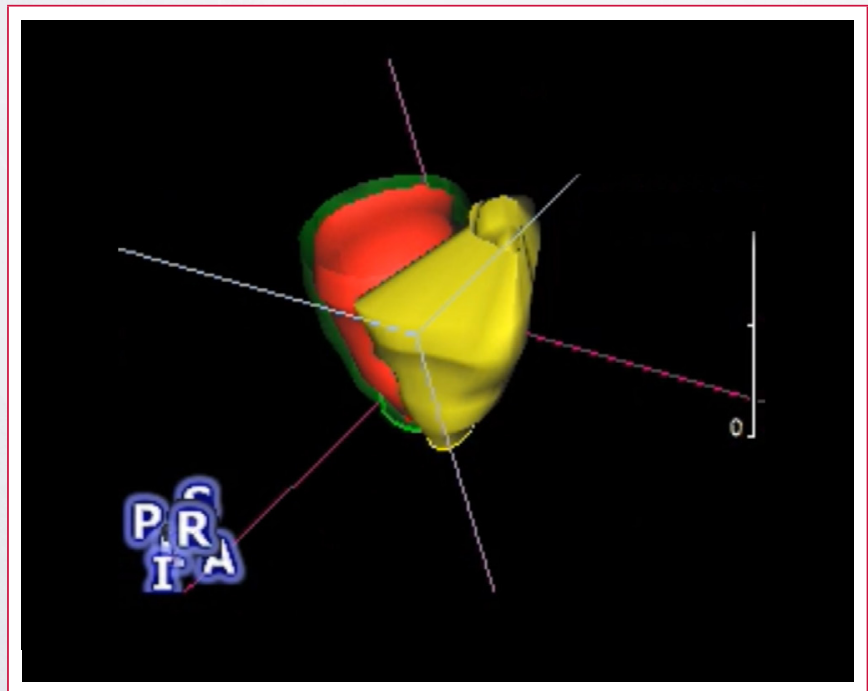

Vídeo 1. Volume rendering telediastólico del ventrículo izquierdo (rojo endocardio, verde epicardio) y del ventrículo derecho (amarillo) 
Aparte de su morfología característica, el VD tiene estructuras anatómicas específicas, que nos van a permitir diferenciarlo del VI: 1) la banda moderadora o trabécula septomarginal, que se extiende a través del VD desde el tabique interventricular hasta la cara anterior de la pared libre, 2) la implantación más apical de la valva septal tricúspide con respecto a la válvula mitral y, por último, 3) las trabeculaciones más acentuadas, que se encuentran en el cuerpo y ápex del VD (Vídeo 2).

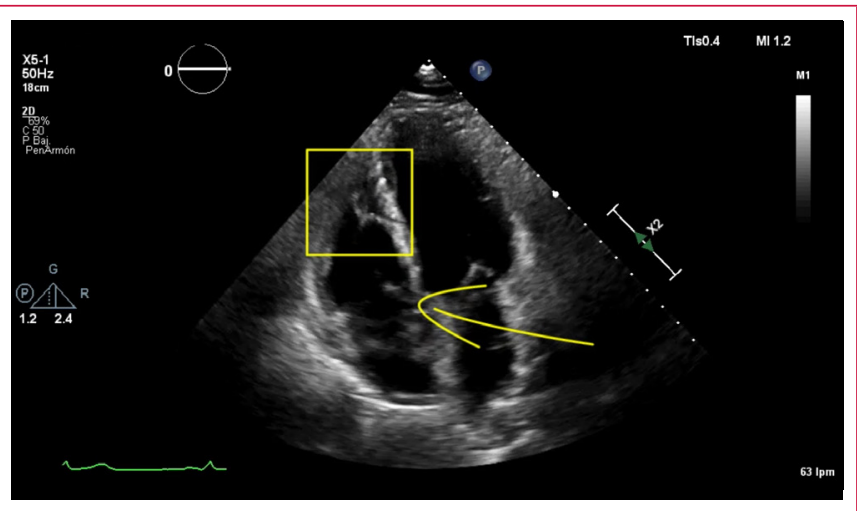

Vídeo 2. Plano apical 4 cámaras. El cuadro amarillo señala la banda moderadora y la flecha amarilla el plano valvular tricúspide

Podemos diferenciar tres zonas anatómicas dentro del VD: el tracto de entrada, el componente trabecular (cuerpo y ápex) y el tracto de salida (Figura 1).

El tracto de entrada tiene una superficie trabeculada y está formado por las paredes lateral, anterior, posterior y el tabique interventricular. En la base del tracto de entrada del VD se encuentra válvula tricúspide, que está compuesta por un anillo y tres velos (septal, anterior y posterior). El aparato subvalvular tricúspide está compuesto por el músculo papilar anterior y los músculos papilares posteriores y septales.

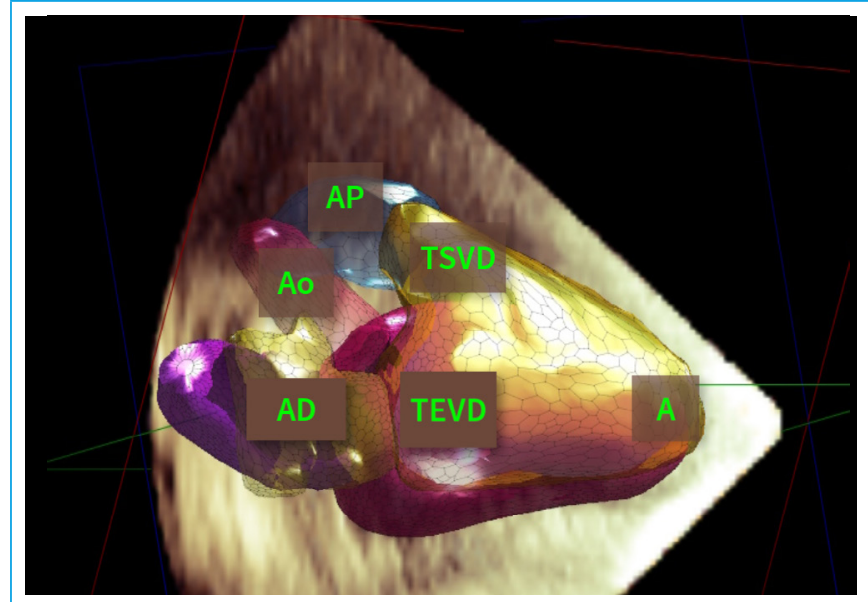

Figura 1. Zoom de una imagen tridimensional, vista desde el lado derecho del corazón, de las cavidades cardiacas (Philips Heart-Model). A: ápex del ventrículo derecho; AD: aurícula derecha; Ao: aorta; izquierda; AP: arterial pulmonar; VD: ventrículo derecho; TEVD: tracto de entrada del ventrículo derecho; TSVD: tracto de salida del ventrículo derecho

El componente apical trabecular incluye el cuerpo y el ápex del VD y se caracteriza por marcadas trabeculaciones.

El tracto de salida -también llamado cono arterial o infundíbulo- es de superficie lisa y se extiende hasta la válvula pulmonar. La cresta supraventricular es una estructura anatómica separa el tracto de entrada del tracto de salida. La cresta supraventricular se extiende desde el anillo tricúspideo y el septo interventricular a través de la banda moderadora hasta la cara anterior del ventrículo derecho (Figura 2).

La aurícula derecha (AD) forma el borde cardíaco derecho, que se encuentra en la parte superior, posterior y derecha del VD. En la AD drena la vena cava su-

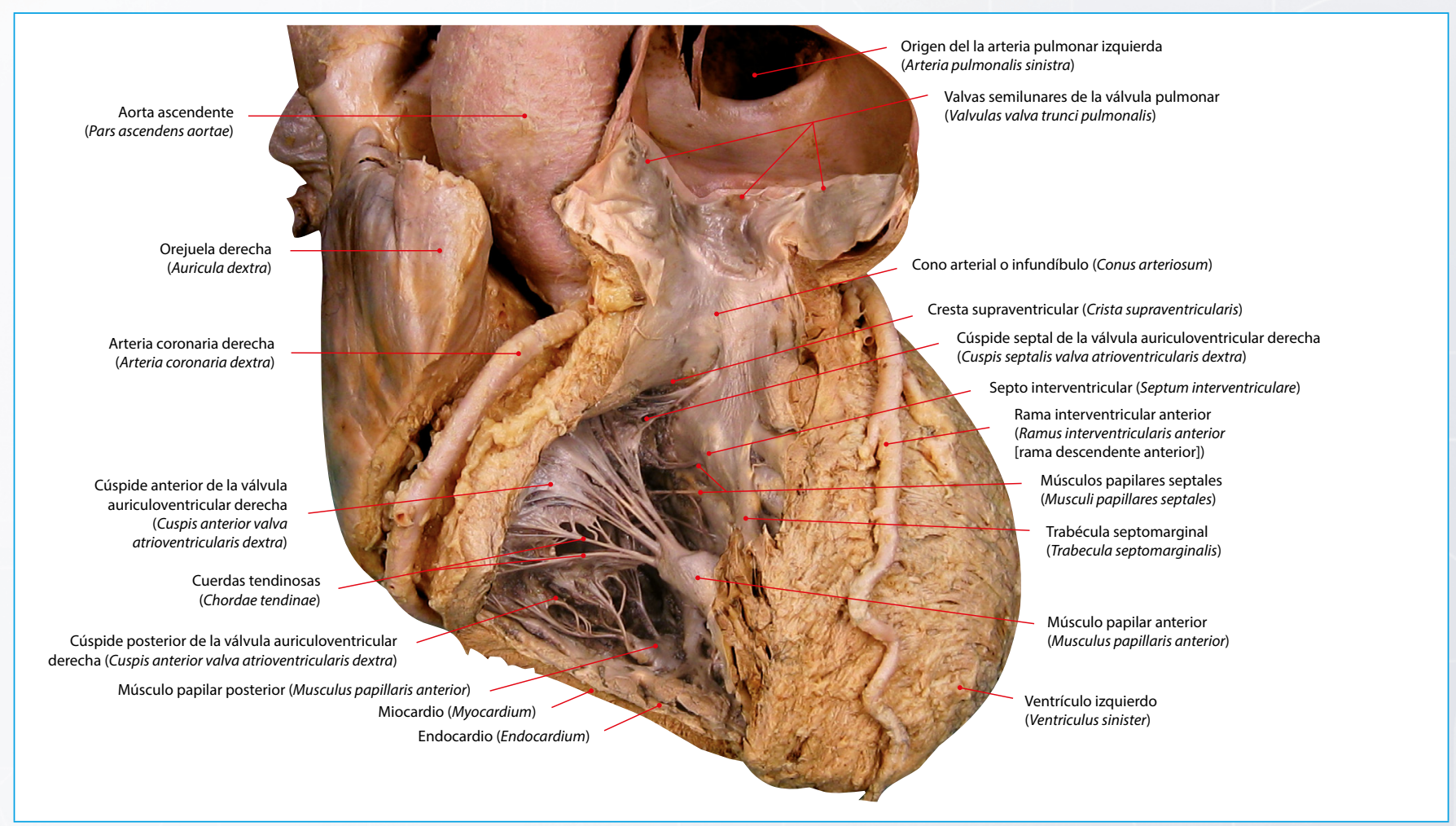

Figura 2. Corazón, visión oblicua anterior (se ha resecado la pared anterior del ventrículo derecho y se ha abierto como un libro la válvula pulmonar) 
perior, en su parte superior y posterior, la vena cava inferior, en su parte inferior y el seno coronario. El orificio del seno coronario está localizado entre la vena cava inferior y el anillo de la válvula tricúspide. El tabique interauricular forma la pared posteromedial de la AD y contiene la fosa oval.

En cuanto a la circulación coronaria, la arteria coronaria derecha es la principal coronaria encargada del suministro arterial de oxígeno al VD. Sin embargo, encontramos diferentes arterias y ramas del árbol coronario izquierdo que también intervienen en el suministro de oxígeno del VD. De esta manera, la arteria descendente anterior izquierda suministra flujo de sangre al VD a través de la arteria de banda moderadora -que surge de la primera rama septal- y de la arteria del cono -que irriga el tracto de salida del VD junto con su arteria homónima que se orgina en la coronaria derecha-. La arteria circunfleja con sus ramas posterolaterales pueden suministrar oxígeno a la parte posterior de la pared libre del VD y la arteria del cono suministran el flujo de sangre al infundíbulo del VD.

\section{Fisiología del ventrículo derecho}

EI VD forma parte de la circulación pulmonar, bombeando sangre desoxigenada proveniente del organismo a los pulmones para que se realice un adecuado intercambio gaseoso. A pesar de una tener una menor masa miocárdica del VD, el gasto cardíaco es el mismo que el del VI. Esto es posible porque la circulación pulmonar es de menor tamaño y de menor resistencia que la circulación sistémica.

La contracción del VD se inicia en la región de entrada del VD, seguida de la región del cuerpo. La mayor parte (hasta el 75\%) de su función sistólica depende una adecuada contracción en plano longitudinal, mientras que una fracción más pequeña está relacionada con la contracción radial o transversal de la pared libre del VD. Este patrón específico de contractilidad de VD y su anatomía hacen que sea de más compleja su evaluación por ecocardiograma como veremos más adelante.

La función diastólica del VD también difiere de la del VI, sus paredes son más finas y, por lo tanto, tienen una distensibilidad mayor que el VI. Esto, permite cambios de volumen mayores sin que la presión diastólica varíe'.

Ambos ventrículos se relacionan entre otras estructuras anatómicas mediante el septo interventricular (SIV). Situaciones clínicas específicas como la hipertensión pulmonar (HTP) producen una sobrecarga de presión de cavidades derechas mientras que algunas cardiopatías congénitas o valvulopatías conllevan una sobrecarga de volumen del VD. En ambos casos se provoca una alteración en la interdependencia interventricular que puede afectar al Ilenado del VI. Cuanto menor es la diferencia de presiones entre ambos ventrículos (sobrecarga de presión en VD), el SIV se aplana adquiere una forma aplanada en diástole, de modo que el VI adquiere forma de "D" en el eje corto (Vídeo 3).

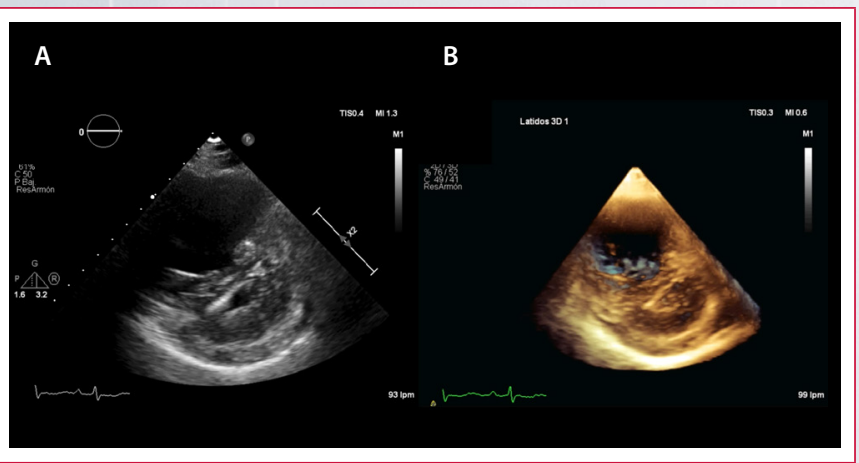

Vídeo 3. Paciente con hipertensión pulmonar s severa. Signo de la D.

A: eje corto 2D. B: eje corto 3D
Este signo de sobrecarga de presión de cavidades derechas se magnifica en situaciones de presión arterial sistémica baja y por el contrario podría podría incluso estar ausente si la presión sistémica aumenta, aunque la HTP sea significativa. La interdependencia ventricular y la morfología del SIV pueden ser cuantificarse por distintos métodos entre otros mediante el índice de excentricidad ${ }^{2}$.

\section{El ecocardiograma en el ventrículo derecho}

Debido a la compleja estructura y geometría del VD, para su completa visualización se necesitan diferentes proyecciones y, en muchas ocasiones, de ventanas complementarias enfocadas al VD.

El plano más utilizado para evaluar de forma inicial la geometría, el tamaño y la función del VD es el apical 4 cámaras (4C) o el 4 cámaras medioesofágico obtenido mediante ecocardiografía transesofágica (ETE) (Vídeo 4). Además del ventrículo derecho es importante analizar otras estructuras cercanas: la aurícula derecha, la vena cava inferior y la válvula tricúspide. Desde la ventana apical 4C, se visualiza de forma adecuada la pared libre o lateral del VD y el SIV, por lo que nos permite cuantificar los diámetros de la cavidad del VD y el grosor de la pared libre de VD (aunque el grosor de la pared libre se estima con más precisión desde la ventana subcostal). El tamaño de la aurícula derecha y la morfología septo interauricular, nos ayudan a evaluar de forma indirecta la presencia de elevación de presiones intracavitarias. En cuanto a la válvula tricúspide, podemos observar el jet de insuficiencia tricúspide, cuya velocidad nos va a permitir estimar la presión pulmonar como veremos en destalle más adelante.

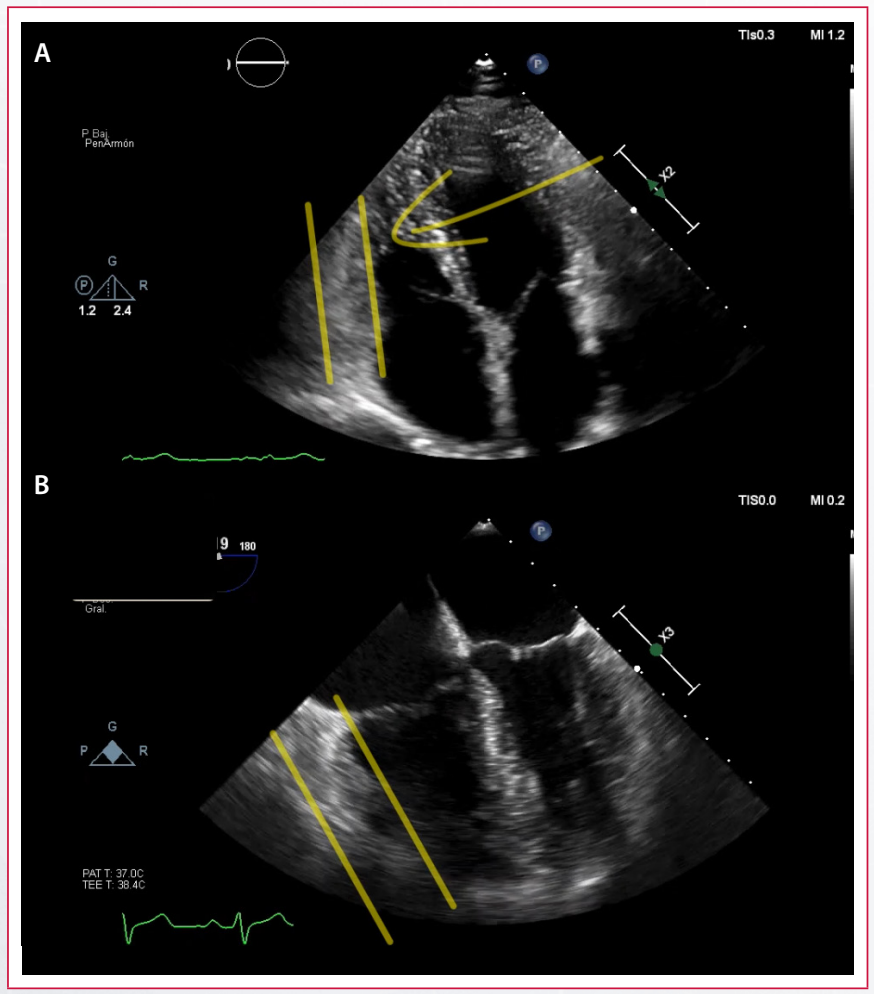

Vídeo 4. A: apical 4 cámaras. B: 4 cámaras ETE plano medioesofágico. Las líneas rectas amarillas destacan la pared lateral del ventrículo derecho. La flecha amarilla señala al hipertrabeculación característica del ventrículo derecho. El plano apical 4 cámaras muestra un ventrículo derecho de morfología y función sistólica normales. El planos 4 cámaras de ETE muestra un ventrículo derecho ligeramente dilatado (el paciente presentaba una comunicación de pequeño tamaño). 
Desde el apical 4C podemos angular el transductor para obtener el plano apical 4C focalizado en VD. Inicialmente, con una ligera angulación a la derecha se centra la visión en la pared anterolateral del ventrículo izquierdo en lugar del ápex, lo que permite la visualización completa de toda la pared libre del $\mathrm{VD}$, convirtiéndolo por tanto en el mejor plano para realizar las mediciones del VD -tanto para evaluar las dimensiones del mismo como su función sistólica-. Si desplazamos el transductor a la derecha del paciente desde el apical $4 C$ obtenemos el plano apical modificado del ventrículo derecho que desde el cual también obtenemos una mejor visualización del VD. En esta posición se tiende a disminuir la longitud del VD (acortamiento longuitudinal) y, por lo tanto, no es una proyección útil para realizar mediciones con precisión (Vídeo 5). Sin embargo este plano en ocasiones es útil para evaluar defectos del septo interauricular y para obtener una mejor alineación del Doppler continuo, para poder estimar la presión arterial pulmonar.

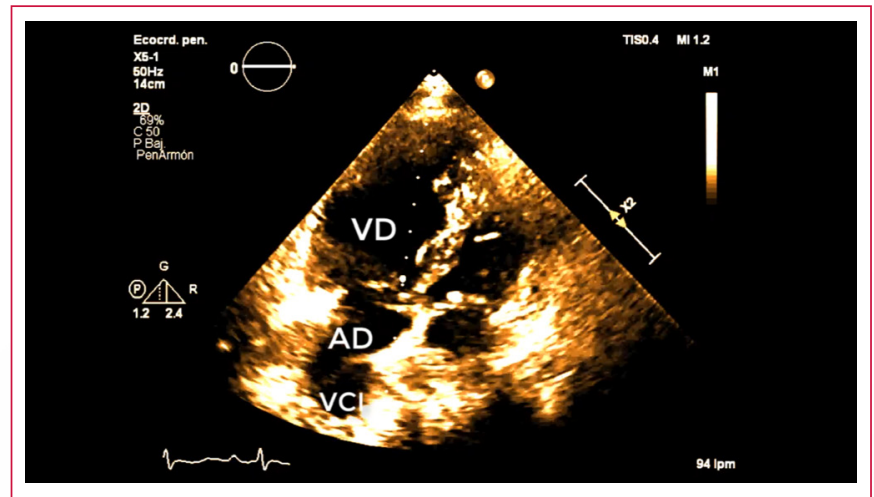

Vídeo 5. Plano apical modificado del ventrículo derecho en un paciente con HTP pulmonar severa. El ventrículo derecho esta severamente dilatado y su fracción de eyección está severamente deprimida. AD: aurícula derecha. VCl: vena cava inferior. VD: ventrículo derecho

En el plano paraesternal eje largo (PEL), observamos principalmente el cuerpo del VD (Vídeo 6).

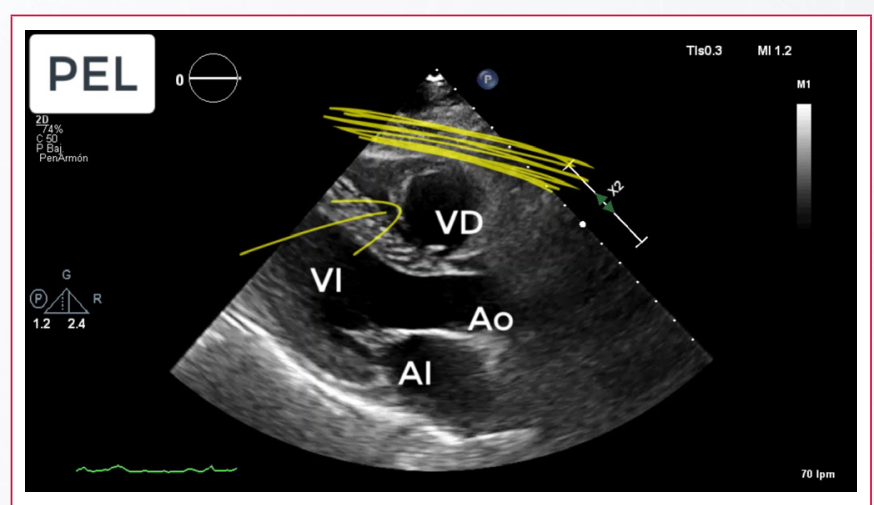

Vídeo 6. Plano paraesternal eje largo. Al: aurícula izquierda. Ao: Aorta. VD: ventrículo derecho. Vl: ventrículo izquierdo. El subrayado-tachado amarillo destaca la pared del ventrículo derecho que se observa en esta proyección: cara anterior. La flecha amarilla señala la banda moderadora

Si realizamos una angulación medial e interna con el transductor conseguimos el plano paraesternal modificado o de tracto de entrada del ventrículo derecho, que permite evaluar el tracto de entrada de VD - incluyendo la aurícula derecha, la válvula tricúspide y el VD-. Desde este plano, se puede evaluar también anatómica y funcionalmente la válvula tricúspide. A nivel anatómico podemos observar la morfología de los velos anterior y posterior (el posterior únicamente es visualizado desde este plano) de la válvula tricúspide y, a nivel funcional, la presencia y los mecanismos de regurgitación (Vídeo 7).

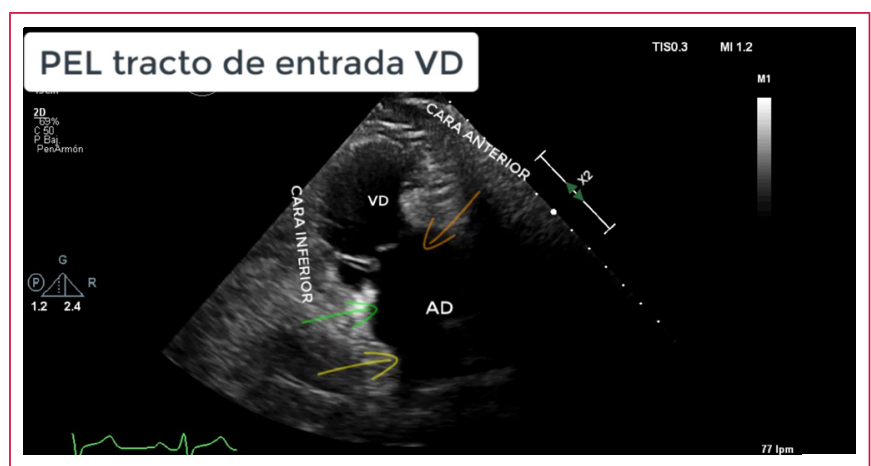

Vídeo 7. Tracto de entrada del ventrículo derecho. AD: aurícula derecha. VD: ventrículo derecho. Flecha verde: valva posterior tricúspide. Flecha naranja: valva anterior tricúspide. Flecha amarilla: valva de Eustaquio. Se observan la cara anterior e inferior del ventrículo derecho

Si desde este plano hacemos una angulación anterior y superior del transductor, encontramos el plano paraesternal de tracto de salida del ventrículo derecho (TSVD) donde podemos evaluar la válvula pulmonar y la arteria pulmonar (Vídeo 8).

La presencia y velocidad de la regurgitación pulmonar nos va a permitir estimar la presión pulmonar y resistencias pulmonares como veremos más adelante. Además, el Doppler pulsado del TSVD es importante para cuantificar la ITV como marcador de volumen sistólico del VD.

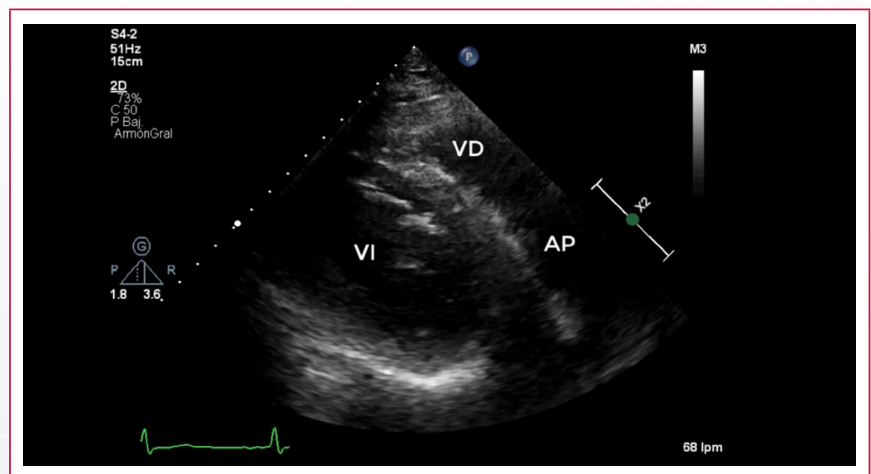

Vídeo 8. Plano paraesternal eje largo TSVD y AP. AP: arteria pulmonar VD: ventrículo derecho. Vl: ventrículo izquierdo

En el plano paraesternal eje corto (PEC), cuando estamos alineados con los músculos papilares del VI, se observa al VD "abrazando" al VI (Vídeo 9). Desde este plano podemos apreciar la posición del septo y la interdependencia interventriculares. No obstante, debido a la asimetría del VD, desde este plano no es posible evaluar correctamente el tamaño ni la función sistólica del VD.

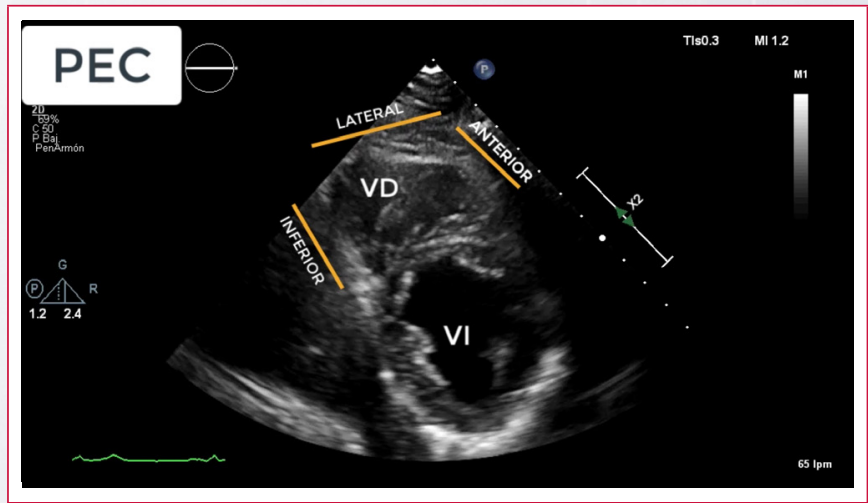

Vídeo 9. Plano paraesternal eje corto. VD: ventrículo derecho. VI: ventrículo izquierdo. Se observan la cara anterior, lateral e inferior del ventrículo derecho 
Si desde este plano, angulamos el transductor para conseguir un plano más anterior y superior, obtenemos el plano paraesternal eje corto de la base o plano de los grandes vasos, un eje corto de la base del corazón con la válvula aórtica en posición central, rodeada por la aurícula izquierda, septo interauricular, válvula tricúspide y TSVD con la válvula y arteria pulmonares (Vídeo 10). Desde esta ventana se puede evaluar mediante Doppler el funcionamiento de las válvulas tricúspide y pulmonar. Desde este plano, podemos observar la presencia de defectos atriales que puedan explicar cambios en el volumen y función del VD.

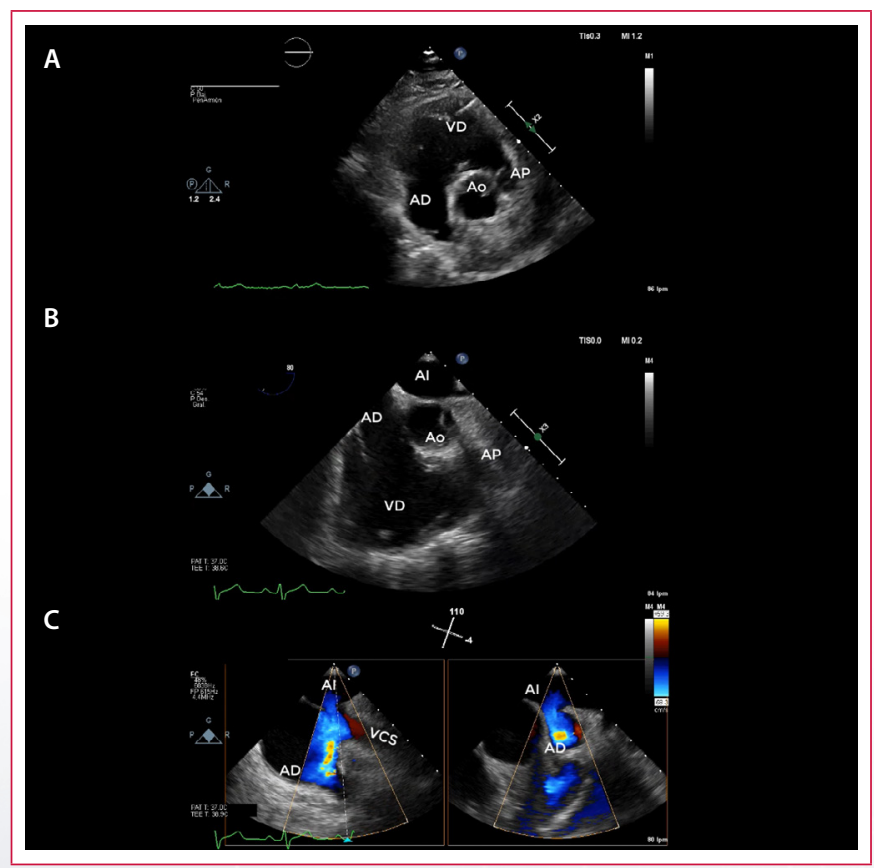

Vídeo 10. A. Plano paraesternal eje corto basal ETT. B. Plano eje corto basal ETE. C. Plano bicavo obtenido mediante ETE. El video B y el C corresponden al mismo paciente. AD: aurícula derecha. Al: aurícula izquierda. Ao: aorta. AP: arteria pulmonar. VCS: vena cava superior. Círculo naranja valva lateral tricúspide. Círculo amarillo valva septal tricúspide. Circulo azul: defecto del septo interauricular (CIA) en la parte superior del tabique interauricular. Este defecto no se observaba en el plano de eje corto porque estaba una porción superior del tabique.

Por último, desde la ventana subcostal el plano subcostal es un plano de gran utilidad para evaluar el grosor de la pared libre del VD, siendo menos fiable para el análisis y medida de las dimensiones intracavitarias y función sistólica en la población adulta ${ }^{1-2}$

\section{Evaluación del ventrículo derecho}

Dimensiones

\begin{tabular}{|l|c|c|}
\hline \multicolumn{1}{|c|}{ Parámetro } & Media \pm DE & $\begin{array}{c}\text { Rango } \\
\text { normalidad }\end{array}$ \\
\hline Diámetro basal del VD $(\mathrm{mm})$ & $33 \pm 4$ & $25-41$ \\
\hline Diámetro medio del VD $(\mathrm{mm})$ & $27 \pm 4$ & $19-35$ \\
\hline Diámetro longitudinal del VD $(\mathrm{mm})$ & $71 \pm 6$ & $59-83$ \\
\hline Diámetro TSVD PEL $(\mathrm{mm})$ & $25 \pm 2.5$ & $20-30$ \\
\hline Diámetro TSVD proximal PEC $(\mathrm{mm})$ & $28 \pm 3,5$ & $21-35$ \\
\hline Diámetro TSVD distal PEC $(\mathrm{mm})$ & $22 \pm 2,5$ & $17-27$ \\
\hline $\begin{array}{l}\text { DE: derivación estándar; VD: ventrículo derecho; PEC: paraesternal eje corto; } \\
\text { PEL: paraesternal eje largo; TSVD: tracto de salida del ventrículo derecho }\end{array}$ \\
\hline
\end{tabular}

Tabla 1. Valores de referencia para el tamaño del ventrículo derecho
- Ventrículo derecho: para medir adecuadamente el VD es preferible el plano apical 4C "focalizado" para VD en telediástole, manteniendo siempre una visualización del ápex verdadero para asegurarnos de no acortar longitudinalmente el VD. Se puede usar contraste intravenoso) para una mejor delimitación del borde endocárdico. Es importante no incluir las trabeculaciones en las mediciones.1-2, 5-6

Diámetros de VD (Figura 3): podemos decir que existe dilatación ventricular cuando: Diámetro basal > 42 mm, diámetro a nivel medio > 35 $\mathrm{mm}$, diámetro longitudinal $>86 \mathrm{~mm}$.

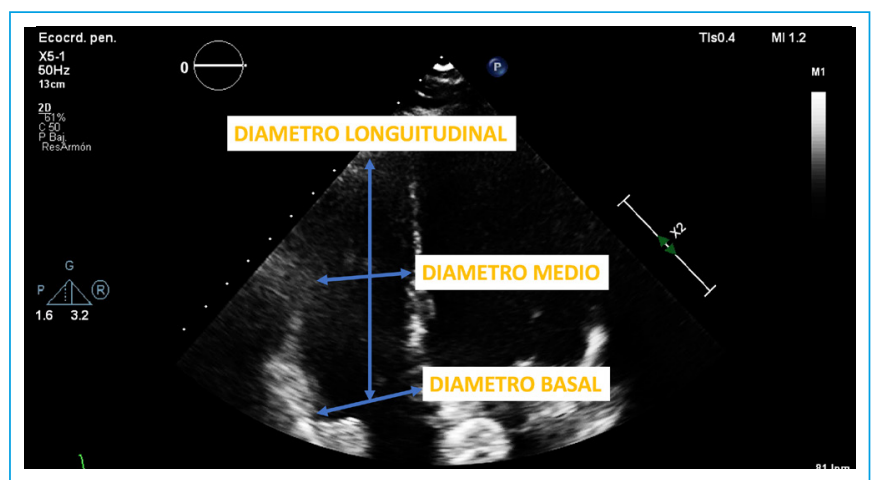

Figura 3. Plano apical 4 cámaras focalizado sobre la cavidad ventricular: diámetros ventriculares.

El área del VD bajo condiciones normales debería ser igual o menor a dos tercios la del VI (Ratio 0.66). Por encima de esta ratio, podemos decir que existe dilatación del VD (leve: 0,7-1: moderado: 1-1,5 y severo > 1,5). Estamos hablando del área en este plano no del volumen del VD, que tiende a ser ligeramente superior como ya se ha comentado.

El volumen de VD es difícil de evaluar por su forma asimétrica y la incapacidad para visualizar la totalidad del VD en una única proyección. La ecocardiografía 3D permite la obtención de unos volúmenes de VD más exactos y fiables. De forma cualitativa en el apical 4C en 2D, el ápex de VD puede aparecer redondeado si el VD está aumentado de tamaño y puede desplazar al VI formando el verdadero ápex si la dilatación es severa.

La hipertrofia del VD medida en telediástole, preferentemente desde el plano subcostal o en el plano apical 4C "focalizado" para VD y teniendo precaución de no incluir la trabeculación del VD, está definida como un grosor de la pared superior a $5 \mathrm{~mm}$. (Vídeo 11).

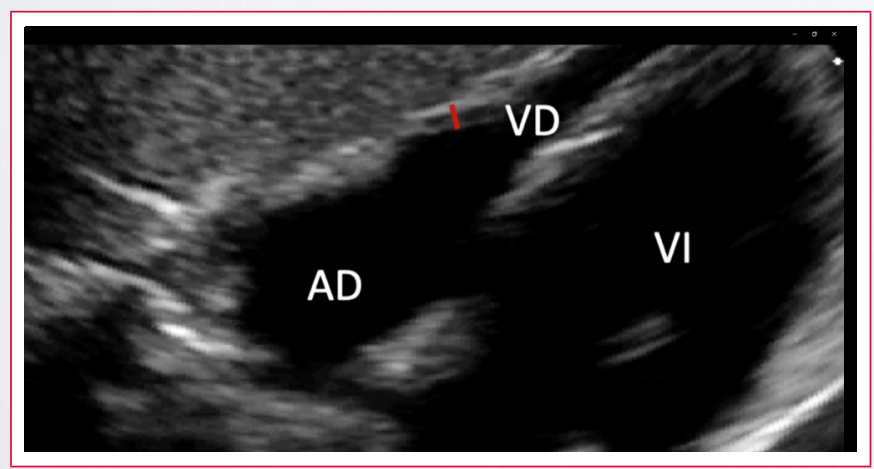

Vídeo 11. Plano subxifoideo 4 cámaras. Para obtener una adecuada medición del espesor de la pared lateral del ventrículo derecho debemos hacer un zoom sobre la imagen en diástole. AD: aurícula derecha. Al: aurícula izquierda. VD: ventrículo derecho. Vl: ventrículo izquierdo. Línea roja: espesor de la pared lateral del ventrículo derecho.

- Aurícula derecha (AD): En el plano 4C se puede medir tanto su área como su diámetro. Podemos decir que existe dilatación de Al si: área $>18 \mathrm{~cm}^{2}$, diámetro longuitudinal $>53 \mathrm{~mm}$, diámetro transversal $>44 \mathrm{~mm}$. 
- TSVD: En el PEC encontramos el TSVD a nivel de la válvula pulmonar. Un diámetro >27 mm en telediástole a nivel de inserción de la válvula pulmonar indica dilatación del TSVD (Figura 4).

- Vena cava inferior (VCI): desde el plano subcostal, se mide el diámetro proximal y porcentaje de colapso en inspiración. De este modo podemos estimar la presión en la aurícula derecha y por lo tanto va a mejorar la estimación de la presión sistólica de la arteria pulmonar (PSAP):

- Diámetro $\mathrm{VCI} \leq 21 \mathrm{~mm}$ con colapso >50\%: Presión de AD normal (0-5 $\mathrm{mmHg}$, media $3 \mathrm{mmHg}$ )

- Diámetro $>21 \mathrm{~mm}$ con colapso < 50\% con la inspiración: elevación de presiones de AD (10-20 mmHg, media $15 \mathrm{mmHg}$ )

- En valores intermedios, situaciones en las que sólo se observe uno de los hallazgos previamente descritos podemos utilizar un valor intermedio de 8 $\mathrm{mmHg}$ (entre 5-10 mmHg)

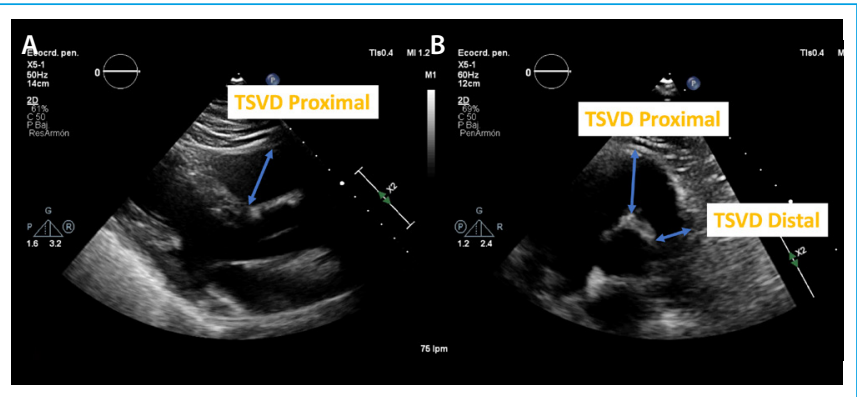

Figura 4. A: plano paraestenal eje largo. B: plano pararesternal eje corto basal. Donde hay que obtener las distintas medidas del tracto de salida del ventrículo derecho (TSVD).

La presión positiva de la ventilación mecánica altera la relación entre la presión venosa central y el tamaño de la VCI por lo que esta estimación no es útil en estos pacientes.

\section{Función sistólica de ventrículo derecho}

Debido a la complejidad anatómica y los diferentes patrones contráctiles del VD, existen múltiples métodos cuantitativos para evaluar la función sistólica del VD como vamos a ver a continuación. Por estos motivos, como normal general, podemos afirmar que, los métodos bidimesionales para calcular los volúmenes y fracción de eyección del ventrículo derecho, área longitud y método Simpson, no se deben aplicar porque como sabemos la morfología del VD es muy diferente al VI. En otras palabras, las asunciones geometrícas del VI no se deberían aplicar al VD.

\section{Medidas de función global del VD}

- FE 3D: El análisis tridimentsioneal de la FE se puede realizar desde un abordaje transtorácico como transesofágico. Es la técnica de referencia para realizar mediciones ecocardiográficas del VD. Los límites superiores de la normalidad de los volúmenes del VD son: $89 \mathrm{ml} / \mathrm{m}^{2}$ para el VTD-VD y $45 \mathrm{ml} /$ $\mathrm{m}^{2}$ para el VTS-VD. El limite por debajo del cual se consideraría que existe disfunción ventricular es una FEVD $<44 \%$. Las principales limitaciones de esta técnica son por un lado el tiempo que se requiere de procesamiento y análisis de las imágenes y por otro que precisa de una adecuada ventana ecocadiográfica 3,1.

- IPMVD (Índice Performance Miocárdico del Ventrículo Derecho o índice de Tei): es un índice de función global del VD (sistólica y diastólica), que viene determinado por la siguiente fórmula (IMPVD $=(\mathrm{TCl}+\mathrm{TRI}) / \mathrm{TE})$, donde TCI es el tiempo de contracción isovolumétrica, TRI es el tiempo de relajación isovolumétrica y TE el tiempo de eyección. Estos tiempos pueden ser determinados mediante Doppler pulsado o Doppler tisular, presentando diferentes valores de referencia según la técnica utilizada. Un IPMVD > 0,44 por Doppler pulsado o > 0,55 por Doppler tisular sugieren disfunción del ventrículo derecho. En caso de aumento de la presión de la $A D$, se acorta el TRI y puede disminuir falsamente el valor de IMPVD.

- FAC (acortamiento fraccional de área): evalúa la función sistólica global del VD y se determina a partir de la diferencia entre las áreas del VD en telediástole y telesístole (FAC = (Área telediástole - Área telesístole) $\times 100 /$ Área telediástole) obtenidas en el plano 4 cámaras "focalizado" en el VD (Figura 5). Es de especial importancia una visualización completa del VD para su medición, sin incluir las trabéculas. Se considera por 2D disfunción sistólica global de VD cuando el FAC es inferior del 35\%.

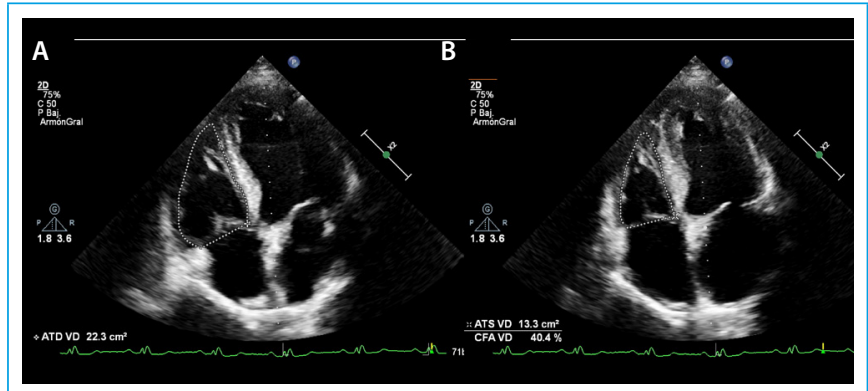

Figura 5. Plano apical modificado del ventrículo derecho en un paciente con HTP pulmonar severa. El ventrículo derecho esta severamente dilatado y su fracción de eyección está severamente deprimida. AD: aurícula derecha. VCl: vena cava inferior. VD: ventrículo derecho

- VD dP/dT: Conforme la contractilidad del VD empeora, el incremento de presión con respecto al paso del tiempo (dP/dT) también disminuye, por lo que es un marcador de deterioro de función sistólica. Puede estimarse de forma no invasiva mediante la obtención de la curva de Doppler continuo de regurgitación tricúspide. Se calcula midiendo el tiempo que requiere la IT para aumentar la velocidad de 1 a $2 \mathrm{~m} / \mathrm{s}$, utilizando la ecuación simplificada de Bernoulli. Aunque dP/dT está libre de asunciones geométricas, requiere una un adecuado alineamiento con la regurgitación tricúspide y una velocidad de barrido del Doppler continuo alta, lo que falicilita la medición del tiempo de aceleración. Los datos actuales sugieren el valor de 400 $\mathrm{mmHg} / \mathrm{seg}$ como límite inferior para la normalidad. ${ }^{1-2}$

\section{Medidas de acortamiento longitudinal de la pared lateral del VD}

- EI TAPSE (desplazamiento longitudinal del anillo tricúspide) continúa siendo una forma habitual y sencilla de cuantificar la función del VD. Aunque únicamente evalúa la contracción longitudinal del VD, ha demostrado una buena correlación con otras medidas que evalúan la función sistólica global. Es una medición que se recomienda realizar de forma rutinaria ya que es fácil de realizar y reproducible. Requiere una adecuada alineación del cursor en modo $\mathrm{M}$ con el anillo tricúspide. El valor de normal del TAPSE es de $24 \pm 3,5 \mathrm{~mm}$. Por tanto cuando el TAPSE es inferior a $16 \mathrm{~mm}$ debe considerarse que la función longitudinal está afectada. En pacientes postoperados cardiacos, el TAPSE normal tiene unos valores de referencia distintos (el valor normal en este subgrupo de pacientes está en torno a $16 \mathrm{~mm}$ ). ${ }^{5,11}$ Es importante destacar que el TAPSE no es una medición fiable para evaluar la función sistólica del pacientes con HTP. En cambio sí se puede utilizar como valor de referencia en el mismo paciente y ver sus variaciones a la largo del tiempo. (Figura 6).

- Onda S' (o velocidad sistólica máxima del anillo tricúspide en su porción lateral) obtenida mediante Doppler tisular. Es una medición que se recomienda realizar de forma rutinaria ya que es fácil de realizar y reproducible. Aunque evalúa la contracción longitudinal del VD, se ha correlacionado de forma adecuada con otros parámetros de función sistólica global del VD. Se obtiene en la proyección apical 4C, situando el cursor del Doppler a nivel del anillo lateral tricúspide o en la zona basal de la pared libre del ventrículo. 
Es importante mantener una buena alineación con la pared lateral del VD. Se considera que el Doppler tisular normal de la onda $S^{\prime}$ es de 14,1 $\pm 2,3 \mathrm{~cm} /$ seg. Cuando obtenemos una onda $S^{\prime}<10 \mathrm{~cm} / \mathrm{s}$ se considera que existe una alteración en la función longuitudinal de la pared libre del VD.11,1

- Strain longitudinal: Es una medida de deformación miocárdica que evalúa anomalías en la contracción del VD sutiles no evaluables por otros métodos. Generalmente, el strain es menor en los segmentos basales y apicales de la pared libre del VD en comparación con el segmento medio. De forma global el Strain de VD es superior al Strain del VI. Se considera un Strain normal de la pared libre del VD por 2D de $-29 \% \pm 3,5 \%$, con un límite de $-20 \%$ para considerar disfunción ${ }^{10}$

\section{Función diastólica}

A pesar de que su análisis no se recomienda de forma rutinaria, la evaluación de la función diastólica puede ser útil para estimar las presiones de llenado de VD o para reconocer estadios iniciales de la afectación del VD cuando todavía la función sistólica está conservada. La disfunción diastólica se relaciona con situaciones clínicas comunes y relevantes como la isquemia de VD, el embolismo pulmonar, HTP y otras condiciones que afectan al VI o ambos ventrículos, como las valvulopatías o el trasplante cardiaco.

De forma similar a la evaluación de la función diastólica del VI, en el plano 4C apical, el Doppler pulsado a través de la válvula tricúspide y el Doppler tisular del anillo lateral tricúspide nos permiten evaluar la relación E/A y E/e'así como tiempo de deceleración de la onda E a través de la válvula tricúspide (valor normal $180 \pm 31 \mathrm{~m} / \mathrm{seg}$ ). Además, también podemos analizar el incremento del flujo diastólico en las venas hepáticas y el aumento de del volumen de la $A D$, que también sugieren disfunción diastólica con elevación de presiones de llenado del VD'.

A la hora de estratificar la severidad de disfunción diastólica, se considera que existe anomalía en la relajación cuando $\mathrm{E} / \mathrm{A}<0,8$. Si E/A 0,8-2,1 con E/e'> 6 o flujo diastólico predominante en las venas hepáticas, sugiere un patrón pseudonormal de relajación. Se considerará un patrón de llenado restrictivo si E/A $>2,1$, con un tiempo de deceleración < 120 mseg. En general, una relación E/e' $>4$ predice una presión de $\mathrm{AD}>10 \mathrm{mmHg}$ con una adecuada sensibilidad y especificidad ${ }^{5,11}$

Es importante destacar que estos parámetros han de ser medidos al final de la espiración y realizar una media de 5 latidos. Los valores obtenidos en caso de taquicardia, arritmia como fibrilación auricular o en casos de regurgitación tricúspide severa pueden no ser válidos ${ }^{1,2}$.

\section{Implicación clínica, limitaciones y líneas de futuro de la técnica}

Actualmente se conoce la importancia pronóstica del tamaño y función del VD en patologías que afectan directamente a las cavidades derechas, como la isquemia del VD, el tromboembolismo pulmonar, la HTP, el SAHS y numerosas cardiopatías congénitas. Sin embargo, también tiene implicaciones pronósticas en patología cardiaca izquierda como: la insuficiencia cardiaca con disfunción ventricular sistólica, en insuficiencia cardiaca con FE preservada y en las valvulopatías izquierdas. El avance en el tratamiento de la insuficiencia cardiaca con dispositivos de asistencia mecánica circulatoria, hace que la adecuada evaluación del VD sea esencial para el éxito de esta terapia. En este sentido, la disminución del pico máximo de Strain longitudinal del VD se ha relacionado el pico máximo de Strain longitudinal de VD con el desarrollo de fracaso deI VD tras la colocación de una asistencia ventricular izquierda. ${ }^{1-4}$
Como hemos visto, existen numerosos métodos cuantitativos para evaluar la función sistólica del VD. La utilización de técnicas en 3D y Strain longitudinal, a pesar ser complejas, tienen una buena correlación con los datos obtenidos por RM cardiaca y son las herramientas más precisas de las que disponemos a día de hoy en ecocardiografía para evaluar correctamente la función del VD. ${ }^{11}$ Como estas técnicas no están aún disponibles en todos los laboratorios todavía, la FAC, el TAPSE y el pico de la onda S son medidas adecuadas y sencillas de obtener y adecuadas para establecer una diagnóstico inicial de la función del VD.

\section{Ideas para recordar}

- EI VD tiene una anatomía y fisiología particular que va a condicionar la metodología para poder evaluarlo.

- Vamos a necesitar varias proyecciones estándar y, en muchas ocasiones, de proyecciones más enfocadas en el VD para un adecuado análisis tanto de su tamaño como de su función.

- La proyección apical 4C "enfocada" en el VD es la mejor proyección para la medición de los diámetros del VD. Para el estudio del TSVD podemos utilizar el plano PEC y el PEL.

- Existen diferentes métodos cuantitativos para la evaluación de la función sistólica. De forma rutinaria se recomienda el cálculo del TAPSE ya que, aunque existen otros métodos más precisos para estimar la función sistólica global del VD también son más complejos de realizar más compleja (FE 3D o Strain longitudinal). La técnica de referencia sigue siendo la cardio-RM.

\section{Abreviaturas}

- $\mathrm{AD}=$ Aurícula derecha

- $\mathrm{Al}=$ Aurícula izquierda

- $\quad \mathrm{ETE}=$ Ecocardiograma transesofágico

- $F A C=$ Acortamiento fraccional de área

- HTP= Hipertensión pulmonar

- $\quad$ ITV = Integral tiempo velocidad

- $\quad$ IPMVD = Indice performance miocárdico del ventrículo derecho o índice de Tei

- $\quad \mathrm{IT}=$ Insuficiencia tricúspide

- PAPd = Presión diastólica de la arteria pulmonar

- PAPm = Presión media de la arteria pulmonar

- PAPs = Presión sistólica de la arteria pulmonar

- $\quad P E L=$ Plano paraesternal eje largo

- $\quad \mathrm{RVP}=$ Resistencias vasculares pulmonares

- $\mathrm{SAHS}=$ Síndrome de apnea-hipopnea del sueño

- $\quad$ SIA = Septo interauricular

- $\quad$ SIV = Septo interventricular

- TAP = Tiempo de aceleración de la arteria pulmonar

- TAPSE = Desplazamiento longitudinal del anillo tricúspide

- $T S V D=$ Tracto de salida del ventrículo derecho

- $\quad \mathrm{VCl}=$ Vena cava inferior

- $V D=$ Ventrículo derecho

- $\mathrm{VI}=$ Ventrículo izquierdo

\section{Bibliografía}

1. Dutta T, Aronow WS. Echocardiographic evaluation of the right ventricle: Clinical implications. Clin Cardiol 2017; 40(8): 542-8.

2. Kaul, Tei C, Hopkins JM, Shah PM. Assessment of right ventricular function using two-dimensional echocardiography. Am Heart J 1984; 107: 526531. 
3. Clark TJ, Sheehan FH, Bolson EL. Characterizing the normal heart using quantitative three-dimensional echocardiography. Physiol Meas 2006; 27: 467-508

4. Rudski LG, Lai WW, Afilalo J, et al. Guidelines for the echocardiographic assessment of the right heart in adults: a report from the American Society of Echocardiography endorsed by the European Association of Echocardiography, a registered branch of the European Society of Cardiology, and the Canadian Society of Echocardiography. J Am Soc Echocardiogr 2010; 23: 685-713.

5. Kovalova S, Necas J, Cerbak R, et al. Echocardiographic volumetry of the right ventricle. Eur J Echocardiogr. 2005; 6: 15-23.

6. Bommer $W$, Weinert $L$, Neumann A, et al. Determination of right atrial and right ventricular size by two-dimensional echocardiography. Circulation 1979; 60: 91-100.

7. Van der Zwaan HB, Helbing WS, McGhie JS, et al. Clinical value of realtime three-dimensional echocardiography for right ventricular quantification in congenital heart disease: validation with cardiac magnetic resonance imaging. J Am Soc Echocardiogr 2010; 23: 134-140.

8. Weidemann F, Eyskens B, Mertens $L$, et al. Quantification of regional left and right ventricular function by ultrasonic strain rate and strain indexes after surgical repair of tetralogy of Fallot. Am J Cardiol. 2002; 90: 133-138.

9. Lang RM, Badano LP, Mor-Avi F, et al. Recommendations for cardiac chamber quantification by echocardiography in adults: an update from the American Society of Echocardiography and the European Association of Cardiovascular Imaging. J Am Soc Echocardiogr 2015; 28: 1-39.

10. Kjaergaard J, Snyder EM, Hassager C, et al. Impact of preload and afterload on global and regional right ventricular function and pressure: a quantitative echocardiography study. J Am Soc Echocardiogr 2006; 19: 515-521.

11. Kukulski T, Hubbert $L$, Arnold M, et al. Normal regional right ventricular function and its change with age: a Doppler myocardial imaging study. J Am Soc Echocardiogr 2000; 13:194-204.

12. Hsiao SH, Lin SK, Wang WC, et al. Severe tricuspid regurgitation shows significant impact in the relationship among peak systolic tricuspid annular velocity, tricuspid annular plane systolic excursion, and right ventricular ejection fraction. J Am Soc Echocardiogr. 2006; 19: 902-910.

13. Ghio S, Recusani F, Klersy C, et al. Prognostic usefulness of the tricuspid annular plane systolic excursion in patients with congestive heart failure secondary to idiopathic or ischemic dilated cardiomyopathy. Am J Cardiol 2000; 85: 837-842.

14. Vivo R, Cordero-Reyes A, Qamar U, et al. Increased right-to-left ventricular diameter ratio is a strong predictor of right ventricular failure after left ventricular assist device. J Heart Lung Transplant 2013; 32:792-799.

15. Goldraich L, Kawajiri H, Foroutan F, et al.: Tricuspid valve annular dilatation as a predictor of right ventricular failure after implantation of a left ventricular assist device. J Cardiol Surg. 2016; 31:110-116. 